\title{
ARTICLE \\ Single and repeated ketamine infusions for reduction of suicidal ideation in treatment-resistant depression
}

\author{
Jennifer L. Phillips ${ }^{1,2}$, Sandhaya Norris ${ }^{1,2}$, Jeanne Talbot ${ }^{1,2}$, Taylor Hatchard ${ }^{1}$, Abigail Ortiz (iD) , Meagan Birmingham ${ }^{1}$, Olabisi Owoeye ${ }^{1,2}$, \\ Lisa A. Batten ${ }^{1}$ and Pierre Blier ${ }^{1,2,3}$
}

\begin{abstract}
Repeated administration of subanesthetic intravenous ketamine may prolong the rapid decrease in suicidal ideation (SI) elicited by single infusions. The purpose of this secondary analysis was to evaluate reduction in SI with a single ketamine infusion compared with an active control, and prolonged suppression of SI with repeated and maintenance infusions. Thirty-seven participants with treatment-resistant depression (TRD) and baseline SI first received a single ketamine infusion during a randomized, double-blind crossover with midazolam. Following relapse of depressive symptoms, participants received six open-label ketamine infusions administered thrice-weekly over 2 weeks. Antidepressant responders ( $\geq 50 \%$ decrease in Montgomery-Åsberg Depression Rating Scale [MADRS] scores) received four further open-label infusions administered once-weekly. Changes in SI were assessed with the suicide items on the MADRS (item 10, MADRS-SI) and the Quick Inventory of Depressive Symptomatology-Self Report (item 12, QIDS-SI). Linear mixed models revealed that compared with midazolam, a single ketamine infusion elicited larger reduction in SI $(P=0.01)$, with maximal effects measured at 7 days postinfusion $(P<0.001$, Cohen's $d=0.83)$. Participants had cumulative reductions in MADRS-SI scores with repeated infusions $(P<0.001)$, and no further change with maintenance infusions $(P=0.94)$. QIDS-SI results were consistent with MADRS-SI. Overall, $69 \%$ of participants had a complete alleviation of SI following repeated infusions. In TRD, single and repeated ketamine infusions resulted in decreases in SI which were maintained with once-weekly maintenance infusions. This study adds to the growing body of research suggesting ketamine as a possible novel treatment strategy for SI in mood disorders.
\end{abstract}

Neuropsychopharmacology (2020) 45:606-612; https://doi.org/10.1038/s41386-019-0570-x

\section{INTRODUCTION}

An estimated 1 million people die from suicide every year, with more than two-thirds of cases occurring during a major depressive episode [1]. Although major depressive disorder (MDD) is amenable to pharmacotherapy, up to one-third of patients fail to respond to treatment [2] and may remain at risk of suicide [3]. Suicidal ideation (SI) is a common symptom in patients with MDD and its development represents a critical step in the progression from suicidal thoughts to attempts [4]. SI often requires rapid intervention(s) but very few treatments are effective in reducing $\mathrm{SI}$ and none are fast-acting [5].

Subanaesthetic doses of intravenous (IV) ketamine, an ionotropic glutamatergic N-methyl-d-aspartate (NMDA) receptor antagonist, have been shown to elicit rapid, albeit transient reduction in depressive symptoms in patients with mood disorders [6]. A recent meta-analysis has also provided evidence of the efficacy of ketamine to acutely decrease SI [7]. It has been postulated that these two therapeutic actions are mediated by an increased transmission at a-amino-3-hydroxy-5-methyl-4-isoxazolepropionic acid (AMPA) receptors [8]. While preliminary evidence of the effects of ketamine on SI primarily came from secondary analyses of randomized controlled trials investigating the antidepressant effects of single ketamine infusions [9-12], several recent trials have examined change in $\mathrm{SI}$ with ketamine as a primary outcome measure [13-15].

Despite the rapid relapse rates following a single ketamine infusion, fewer studies have examined the administration of ketamine beyond an acute treatment phase. While reduction in $\mathrm{SI}$ appears to last at least 7 days following a single infusion [7], data from open-label trials suggest that antisuicidal effects may be sustained with repeated infusions [16-18]. Few studies have examined the development of a maintenance strategy that would allow the beneficial effects of ketamine to be prolonged with reduced frequency of infusions. In a secondary analysis of data derived from a ketamine augmentation trial involving participants with treatment-resistant depression (TRD) and SI, we tested whether the antisuicidal effects of ketamine could be augmented through a course of repeated infusions, and whether once-weekly ketamine administration was sufficient to maintain the attenuation of SI obtained with repeated infusions. We have recently

\footnotetext{
${ }^{1}$ Mood Disorders Research Unit, The Royal's Institute of Mental Health Research, Ottawa, ON, Canada; ${ }^{2}$ Department of Psychiatry, University of Ottawa, Ottawa, ON, Canada and ${ }^{3}$ Department of Cellular and Molecular Medicine, University of Ottawa, Ottawa, ON, Canada Correspondence: Jennifer L. Phillips (Jennifer.Phillips@theroyal.ca)

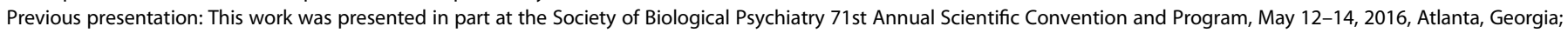
the International College of Neuropsychopharmacology 30th World Congress, July 3-5, 2016, Seoul, South Korea; the 29th European College of Neuropsychopharmacology

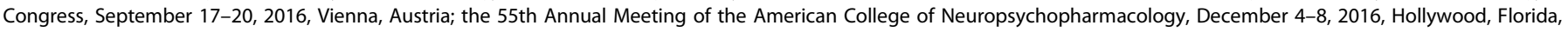
and the 31st International College of Neuropsychopharmacology World Congress, June 16-19, 2018, Vienna, Austria.
}

Received: 12 July 2019 Revised: 23 October 2019 Accepted: 11 November 2019

Published online: 23 November 2019 
shown that this administration strategy extends duration of depressive symptom remission following repeated infusions [19].

A limitation of previous examinations of the antisuicidal effects of ketamine is the inclusion of individuals that are not highly suicidal [7]. Importantly, recent data suggests that ketamine's effects may be less pronounced in individuals with higher SI severity $[20,21]$. Therefore, we also examined the effects of ketamine on SI separately in the subgroup of participants with marked pretreatment SI. Finally, we examined the relationship between ketamine's antidepressant and antisuicidal effects. A recent metaanalysis suggests that reductions in $\mathrm{SI}$ with ketamine are independent of antidepressant effects and not solely the result of a rapid overall decrease in depressive symptoms [7]. This is further supported by findings of reduced $\mathrm{SI}$ even in patients who fail to achieve an antidepressant response to ketamine $[16,18,22]$.

The objectives of this secondary analysis were to (1) demonstrate whether a single infusion of ketamine elicits greater decrease in SI compared with midazolam (an active control); (2) investigate the extent to which repeated ketamine infusions elicit decrease in SI; (3) ascertain whether suppression of SI following a course of repeated infusions can be prolonged with weekly maintenance infusions; and (4) determine whether the antidepressant and antisuicidal effects of ketamine are independent. We hypothesized that a course of repeated ketamine infusions would elicit decrease in SI independent of antidepressant effects and that reduction in SI would be prolonged throughout maintenance treatment.

\section{MATERIALS AND METHODS}

Participants

The data derive from a previously published single-center clinical trial investigating the antidepressant effects of ketamine infusions in participants with TRD at the Royal's Institute of Mental Health Research in Ottawa, Ontario, Canada [19]. Participants were recruited from physician referrals and advertisements between January 2013 and December 2017. Male and female outpatients, aged 18-65 years old, met criteria for MDD based on the Diagnostic and Statistical Manual of Mental Disorders, 4th edition (DSM-IV-TR) [23], confirmed by the Mini-International Neuropsychiatric Interview (MINI) [24]. Inclusion criteria required at least moderate depression severity (Montgomery-Åsberg Depression Rating Scale (MADRS) [25] total score $\geq 25$ ) and evidence of treatment resistance, defined as failure to respond to at least two medications of different pharmacological classes and two augmentation strategies during the present episode using the Antidepressant Treatment History Form (ATHF) [26]. Inclusion in this secondary analysis required presence of baseline SI defined as MADRS suicide item (item 10, MADRS-SI) score $\geq 2$ ("weary of life/ fleeting suicidal thoughts"). A subgroup of individuals were identified as exhibiting marked SI defined as baseline MADRS-SI score $\geq 4$ ("better off dead/suicidal thoughts common"). Exclusion criteria for the study included history of substance use disorder, body mass index $\geq 35$ (required to minimize side effects as ketamine is dosed by weight), and unstable medical conditions. Participants remained on stable dosages of concomitant psychotropic medication with treatment durations of at least 6 weeks throughout the trial. The Royal Ottawa Mental Health Centre Research Ethics Board approved the protocol. All participants provided written informed consent.

Study design

This is a secondary analysis of a three-phase trial designed to assess the antidepressant effects of ketamine in participants with TRD. In Phase 1, participants were administered a single ketamine infusion during a randomized, double-blind crossover with midazolam (a short-acting benzodiazepine that serves as an active control for ketamine) [22]. Infusions of ketamine hydrochloride (Ketalar ${ }^{\circledR}$, ERFA Canada Inc., Montreal, QC; $0.5 \mathrm{mg} /$ $\mathrm{kg}$, diluted in $0.9 \%$ saline) and midazolam $(30 \mu \mathrm{g} / \mathrm{kg}$ diluted in saline) were administered over a 40 min period by IV pump in an outpatient clinic by a study physician and research nurse under cardiorespiratory monitoring. On infusion days, participants held benzodiazepines from the preceding day as they attenuate ketamine response [27]. Each infusion during Phase 1 occurred at least 7 days apart and subjects required a return of $80 \%$ of their baseline MADRS total scores to proceed to the second Phase 1 infusion and to begin Phase 2. In Phase 2, the repeated treatment phase, participants were administered a course of six open-label ketamine infusions thrice-weekly over a 2-week period. In Phase 3, the maintenance phase, participants who obtained an antidepressant response to ketamine following the course of repeated Phase 2 infusions received once-weekly ketamine infusions for a further 4 weeks. Antidepressant response was defined as $\geq 50 \%$ decrease in MADRS total score from baseline (prior to first infusion in Phase 1) to the end of Phase 2. Nonresponders exited the study following Phase 2.

\section{Outcome measures}

Assessment instruments included the 10-item clinician-administered MADRS, and the 16-item patient-administered Quick Inventory of Depressive Symptomatology-Self Report (QIDS-SR) [28]. For this secondary analysis, the primary outcome measure was change in SI following single and repeated ketamine infusions, measured by the MADRS suicide item (MADRS-SI). MADRS-SI has been shown to be particularly sensitive to assess rapid changes in SI as occur with ketamine [29]. Secondary measures included change in patient-reported thoughts of death or suicide (QIDS-SR item 12, QIDS-SI), and change in depressive symptoms (MADRS items 1-9, with item 10, suicidal thoughts, removed from the MADRS total score to reduce redundancy). During Phase 1, ratings for the MADRS were obtained prior to each infusion and at $2 \mathrm{~h}, 24 \mathrm{~h}$, and 7 days postinfusion, while participants completed the QIDS-SR at preinfusion, and at $24 \mathrm{~h}$, 4 days, and 7 days postinfusion. For Phases 2 and 3, follow-up measures were administered 3 days after the final infusion in each phase. MADRS ratings were conducted by study physicians who trained together to establish adequate inter-rater reliability.

\section{Statistical analysis}

For Phase 1, a random-effects linear mixed model was used to evaluate changes in MADRS-SI scores with ketamine and midazolam infusions across four time points from preinfusion to 7 days postinfusion. Included in the model were subject as a random effect, drug and time as fixed within-subject factors, the interaction between drug and time, and a fixed intercept. Baseline MADRS-SI scores and order of drug administration were included as covariates with fixed within- and between-subject effects, respectively. A first order factor analytic-covariance matrix best fit the data using Akaike's information criteria [30]. Significant effects were examined with Bonferroni-corrected simple effects tests. Cohen's $d$ was used to compare the size of effects of ketamine vs. midazolam at each assessed postinfusion time point. For Phases 2 and 3, random-effects models were used to quantify changes in MADRS-SI scores over time adjusting for phase-specific baseline MADRS total scores. Analyses of QIDS-SI data were performed as described above for MADRS data. To assess whether the effects of ketamine on SI were independent of its effects on other depressive symptoms, the Phase 1 and 2 linear mixed models evaluating change in SI with ketamine were repeated with change in depressive symptoms (MADRS items 1-9) added as a covariate. Further, the correlation between change in SI and change in depressive symptoms through the end of Phase 2 was calculated using a Pearson correlation coefficient. All statistical analyses were repeated for the subgroup with marked SI at baseline. Statistical analyses were performed using IBM SPSS Statistics, version 24.0 
(SPSS Inc., Chicago, Illinois). Results were considered significant at a two-tailed $P$ value of 0.05 .

\section{RESULTS}

\section{Participant characteristics}

Participant screening and enrollment details from the original trial have been previously published [19]. Participant disposition for the current secondary analysis are summarized in Fig. S1. Thirtyseven study participants met criteria for baseline $\mathrm{SI}$ and were included in this analysis. Baseline characteristics of participants are summarized in Table 1. Treatment failures are summarized in Table S1. Five participants from the original trial were excluded from the present analysis due to lack of SI at baseline (MADRS-SI $\leq$ 1). Two participants withdrew from the study, one after completing Phase 1, and another after receiving three infusions in Phase 2. Eighteen of the 37 participants met criteria for marked baseline SI and were included in the subgroup analyses.

\section{Single ketamine infusion}

Thirty-seven participants completed the randomized, double-blind crossover comparison of single infusions of ketamine and midazolam. A random-effects linear mixed model analysis adjusted for baseline MADRS-SI scores and order of drug administration revealed significant main effects for drug $\left(F_{1,42}=\right.$ $6.83, P=0.01)$ and time $\left(F_{3,110}=11.28, P<0.001\right)$, and a significant drug-by-time interaction $\left(F_{3,120}=8.86, P<0.001\right)$. Post hoc simple effects tests revealed that participants had lower MADRS-SI scores with ketamine compared with midazolam at $2 \mathrm{~h}(P=0.03$, Cohen's $d=0.44)$, and 7 days postinfusion $(P<0.001$, Cohen's $d=0.83)$ (Fig. 1). MADRS-SI scores did not differ at $24 \mathrm{~h}$ postinfusion $(P=$

Table 1. Baseline demographic and clinical characteristics of study sample.

Variable

Participants, $n$ (\%)

Age, years, Mean (SD)

Sex, M/F, $n(\%)$

Weight, kg, Mean (SD)

Body mass index, $\mathrm{kg} / \mathrm{m}^{2}$, Mean (SD)

Length of current episode, years, Mean (SD)

Major depressive episodes, Single/recurrent, $n \%$

Failed antidepressant trials ${ }^{\mathrm{a}}$, Mean (SD)

Failed antidepressant augmentations ${ }^{\mathrm{a}}$, Mean (SD)

ECT nonresponder in current episode, $n$ (\%)

MADRS total score, Mean (SD)

MADRS-SI score, Mean (SD)

QIDS-SR total score, Mean (SD)

Lifetime history of suicide attempt, $n(\%)$

Comorbid panic disorder, $n(\%)$

Comorbid agoraphobia, $n$ (\%)

Comorbid social phobia, $n$ (\%)

Comorbid obsessive compulsive disorder, $n(\% 0$

Comorbid alcohol dependence, $n$ (\%

Comorbid generalized anxiety disorder, $n$ (\%)

$E C T$ electroconvulsive therapy, $F$ female, $M$ male, MADRS MontgomeryÅsberg Depression Rating Scale [25], MADRS-SI Montgomery-Åsberg Depression Rating Scale suicide item, QIDS-SR Quick Inventory of Depressive Symptomatology-Self Report [28].

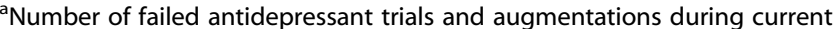
episode according to the Antidepressant Treatment History Form [26]

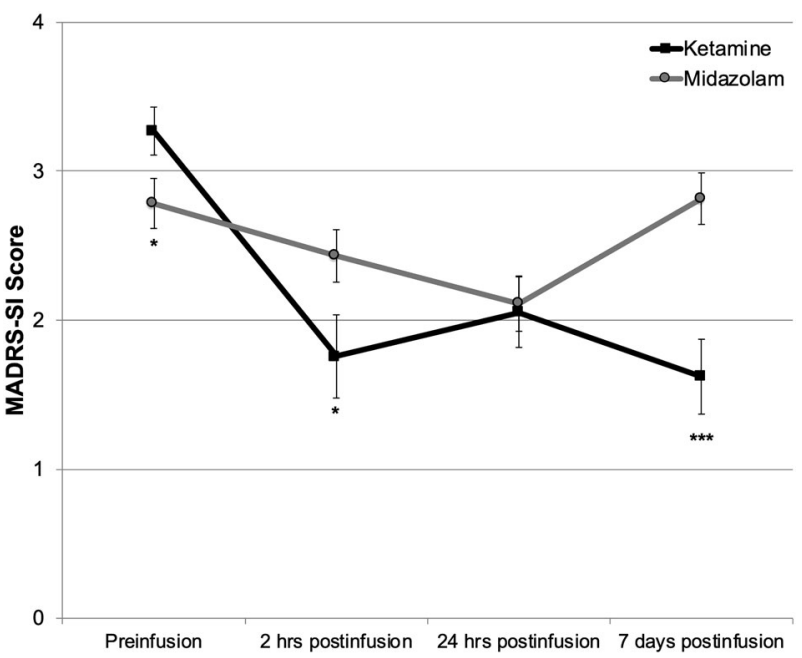

Fig. 1 Change in estimated marginal mean Montgomery-Åsberg Depression Rating Scale suicide item (MADRS-SI) scores over time in patients with treatment-resistant depression treated with single infusions of ketamine and midazolam using a randomized, doubleblind, crossover design $(N=37)$. Error bars represent SEM. Significant difference between ketamine and midazolam, ${ }^{* * *} P<0.001$, ${ }^{*} P<0.01,{ }^{*} P<0.05$.

0.90 , Cohen's $d=0.003$ ). The maximal effect of ketamine on SI was measured at 7 days postinfusion, when participants had an estimated mean decrease in MADRS-SI scores of 1.7 points relative to preinfusion and the estimated mean difference in MADRS-SI scores between ketamine and midazolam was 1.2 points.

Examination of the model covariates revealed significant main effects for baseline MADRS-SI scores $\left(F_{1,48}=130.86, P<0.001\right)$, and for order of drug administration $\left(F_{1,47}=10.17, P=0.003\right)$ on change in $\mathrm{SI}$ during Phase 1. Participants randomized to receive midazolam first had similar preinfusion MADRS-SI scores for both Phase 1 infusions ( $3.3 \pm 1.1$ vs. $3.2 \pm 1.3 ; t_{17}=0.52, P=0.61$ ). Participants randomized to ketamine first had lower preinfusion MADRS-SI scores at the midazolam infusion ( $3.3 \pm 1.3$ vs. $2.3 \pm 1.5$; $\left.t_{18}=3.90, \quad P=0.001\right)$ indicating significant carry-over effects between Phase 1 infusions.

Analysis of change in self-reported $\mathrm{SI}$ during Phase 1 also revealed a significant main effect for drug $\left(F_{1,53}=4.32, P=0.04\right)$, with lower QIDS-SI scores with ketamine relative to midazolam, and a significant main effect for time $\left(F_{3,95}=2.67, P=0.05\right)$. The drug-by-time interaction for QIDS-SI scores was not significant $\left(F_{3,98}=1.89, P=0.14\right)$.

Repeated ketamine infusions

At the start of Phase 2, MADRS-SI scores were significantly lower than at study baseline (prior to the first Phase 1 infusion) $\left(t_{35}=\right.$ $5.87, P<0.001)$. Overall, a 0.9 point decrease in MADRS-SI scores was carried-over from Phase 1 into Phase 2 despite relapse of $97 \%$ of depressive symptoms (mean baseline MADRS items 1-9 scores $32.2[ \pm 3.4]$ vs. pre-Phase 2 scores $31.0[ \pm 3.7])$. Examination of the effects of the course of six repeated ketamine infusions using a random-effects linear mixed model adjusted for subjects and prePhase 2 MADRS-SI scores revealed a significant main effect for time $\left(F_{6,116}=11.86, P<0.001\right)$ (Fig. 2a). Bonferroni-corrected post hoc tests revealed that significant cumulative decreases in MADRS-SI scores relative to the start of Phase 2 became apparent following three successive infusions and for each infusion thereafter. The estimated mean MADRS-SI score for participants at the post-Phase 2 follow-up visit was 1.0. Overall, between baseline and the end of Phase 2, participants had an estimated mean reduction of 2.3 points on the MADRS-SI. 


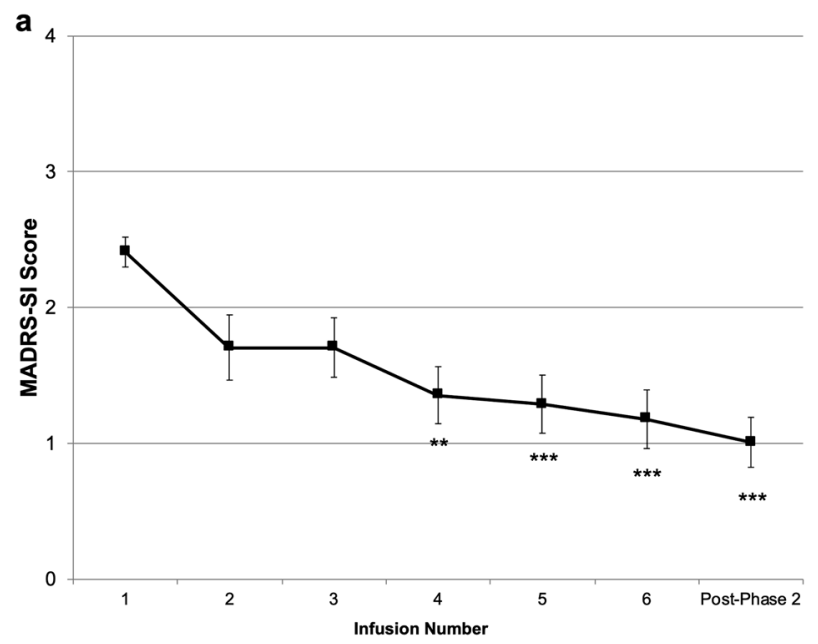

b

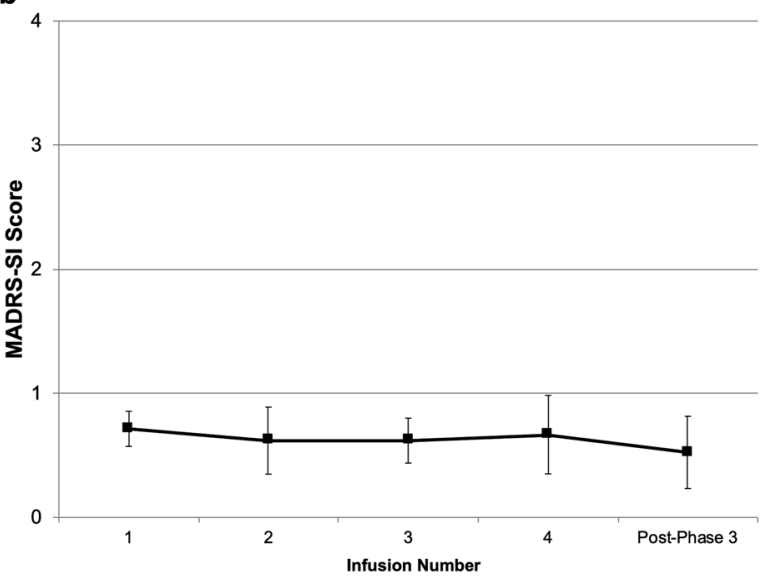

Fig. 2 a Estimated marginal mean preinfusion Montgomery-Åsberg Depression Rating Scale suicide item (MADRS-SI) scores over six open-label repeated ketamine infusions in Phase $2(N=36)$. Error bars represent SEM. Significant difference relative to Phase 2 baseline, ${ }^{* * *} P<0.001,{ }^{*} P<0.01$. b Estimated marginal mean preinfusion Montgomery-Åsberg Depression Rating Scale suicide item (MADRS-SI) scores over four open-label maintenance ketamine infusions in Phase $3(N=21)$. Error bars represent SEM.

There was also a significant main effect of time in the linear mixed model examining change in SI during Phase 2 with the patient-administered QIDS-SI $\left(F_{6,101}=3.76, P=0.002\right)$. Bonferronicorrected post hoc tests revealed that the significant decrease in QIDS-SI scores only became apparent after all six ketamine infusions were administered. Furthermore, there was a significant positive correlation between change in clinician-administered MADRS-SI scores and change in patient-administered QIDS-SI scores during Phase $2(r=0.70, P<0.001)$.

Maintenance ketamine infusions

Within the main trial, participants who achieved an antidepressant response to repeated ketamine infusions $(\geq 50 \%$ reduction in MADRS total scores) continued into the maintenance phase. Twenty-one participants (60\%) included in the current secondary analysis underwent maintenance infusions. A linear mixed model adjusted for subjects and pre-Phase 3 MADRS-SI scores revealed no main effect for time on MADRS-SI scores during maintenance infusions $\left(F_{4,40}=0.19, P=0.94\right)$. This indicates no change in MADRS-SI scores once ketamine infusions were reduced to onceweekly administration (Fig. 2b). Similarly, there was no change in patient-rated QIDS-SI scores during Phase $3\left(\mathrm{~F}_{4,38}=1.74, P=0.16\right)$.

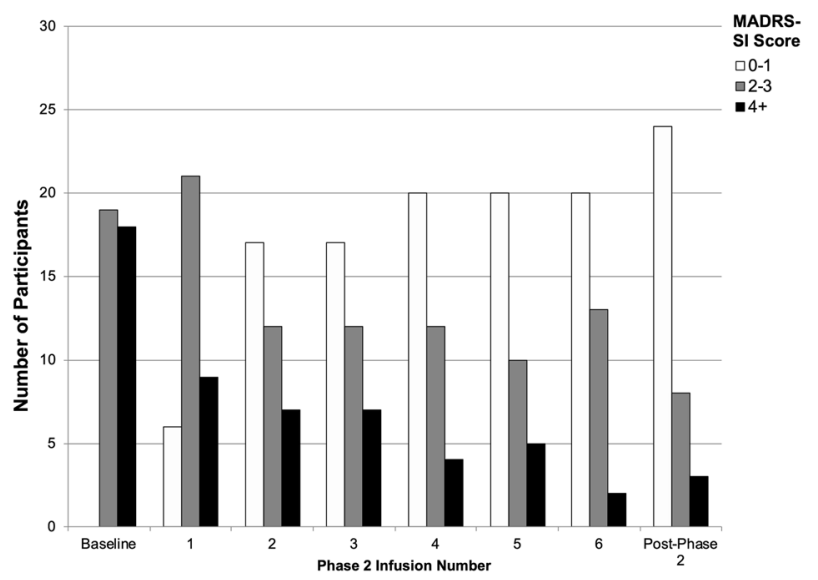

Fig. 3 Distribution of participants' Montgomery-Åsberg Depression Rating Scale suicide item (MADRS-SI) scores at study baseline and over six repeated ketamine infusions in Phase $2(N=37)$.

Alleviation of $\mathrm{SI}$ with repeated infusions

At baseline, prior to randomization in Phase 1, nearly half of the sample (18 participants, 49\%) reported marked SI (MADRS-SI $\geq 4$ ). Following the repeated ketamine infusions administered in Phase 2, 24 participants (69\%) had a lack of SI (MADRS-SI rating of 0 or 1 on the 6-point scale), 8 participants (23\%) endorsed fleeting SI (MADRS-SI $=2)$, and only three individuals remained at ratings at or above 4 (Fig. 3).

Effects of ketamine in individuals with marked SI at baseline Results for the subgroup of 18 participants with marked pretreatment SI (baseline MADRS-SI scores $\geq 4$ ) were similar to those obtained for the full sample. On average, MADRS-SI scores for this subgroup decreased a total of 2.7 points during the trial. Linear mixed models revealed a larger reduction in SI following the single ketamine infusion compared with midazolam $\left(F_{1,19}=11.69\right.$, $P=0.003)$. Thrice-weekly repeated ketamine infusions elicited cumulative decreases in MADRS-SI scores $\left(F_{6,49}=6.61, P<0.001\right)$, with no further change in scores with weekly maintenance infusions $\left(F_{4,14}=0.55, P=0.70\right)$.

Relationship between the antisuicidal and antidepressant effects of ketamine

The relationship between the effects of ketamine on SI and depressive symptoms was examined in the full group using MADRS-SI and MADRS items 1-9 scores, respectively. Participants' change in MADRS-SI scores from baseline to post-Phase 2 were significantly correlated with their change in scores for MADRS items $1-9\left(R^{2}=0.40, r=0.63, P<0.001\right)$ (Fig. 4). However, change in MADRS 1-9 scores only accounted for $40 \%$ of the explained variance in change in MADRS-SI scores, suggesting that the effects of ketamine on SI are not entirely dependent on change in depression severity. Further, when the linear mixed model analyses for single and repeated infusions (Phases 1 and 2) were adjusted for change in depressive symptoms (MADRS items 1-9), most results remained significant. Analysis of the Phase 1 data with the inclusion of change in depressive symptoms as a covariate revealed a significant drug-by-time interaction $\left(\mathrm{F}_{3,117}=\right.$ $9.51, P<0.001)$, and a significant main effect of time $\left(F_{3,160}=4.51\right.$, $P=0.005)$, however the main effect of drug was no longer significant $\left(F_{1,115}=0.39, P=0.54\right)$. This suggests that collapsing across time, the overall decrease in SI with ketamine relative to midazolam is associated with decrease in depressive symptoms, yet the timing of the maximal effects of ketamine on SI and on depression may differ. The antidepressant effects of ketamine were greatest $24 \mathrm{~h}$ postinfusion [19] while the maximal decrease in SI was measured at 7 days postinfusion. In the analysis of the 


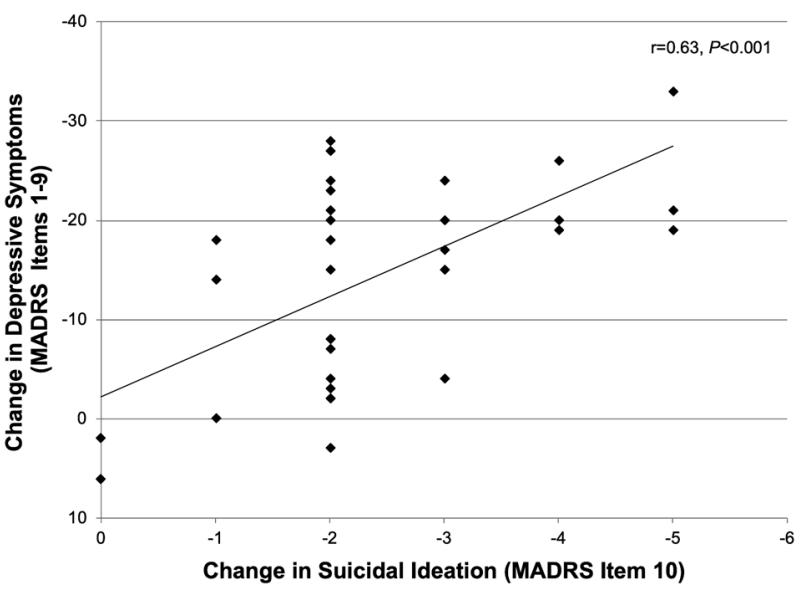

Fig. 4 Correlation between participants' overall change in depressive symptoms (Montgomery-Åsberg Depression Rating Scale, MADRS items 1-9) and their change in suicidal ideation (MADRS suicide item, MADRS-SI) between baseline and post-Phase 2 followup $(N=36)$.

Phase 2 data adjusted for change in scores on MADRS items 1-9, the main effect of time remained significant for the whole group $\left(\mathrm{F}_{6,123}=3.05, P=0.008\right)$, and for the high $\mathrm{SI}$ subgroup $\left(\mathrm{F}_{6,49}=\right.$ $6.61, P<0.001)$. This suggests that the cumulative decrease in $\mathrm{SI}$ with repeated ketamine infusions are not dependent on the antidepressant effects. Indeed, of the 14 antidepressant nonresponders, five participants (36\%) had a complete alleviation of SI following the repeated infusions (post-Phase 2 MADRS-SI $\leq 1$ ).

\section{Safety outcomes}

No serious adverse events were reported during the trial. Vital signs were continuously evaluated at each infusion throughout the study. The most commonly reported side effects associated with ketamine administration were cardiorespiratory effects (transient increases in blood pressure and heart rate), numbness or tingling, dissociation, dizziness, and visual disturbances. Side effects generally resolved within $2 \mathrm{~h}$ of the start of the infusion. Safety outcomes of the trial have been reported in more detail in a previous publication [19].

\section{DISCUSSION}

The findings reported herein support the rapid and cumulative effects of single and repeated ketamine infusions on suicidal thoughts in individuals with TRD, even for those with high pretreatment SI severity. In subjects with an antidepressant response to ketamine, the alleviation of SI obtained following single and repeated infusions was prolonged with once-weekly treatment. The effects of ketamine on SI appear to be at least partially independent of antidepressant effects and were seen even in participants who failed to meet antidepressant response criteria.

All but three participants ( $92 \%$ of the sample) had a decrease in SI following repeated ketamine infusions, including 14 subjects with marked $\mathrm{SI}$ at baseline. These results substantiate emerging evidence that ketamine is a potential therapeutic option for patients with acute SI consistent with previous reports [7]. The three patients without an SI decrease were among those with the highest baseline SI. This finding may be consistent with that of Ballard et al. [20] who found, using pooled data from five clinical trials, that individuals with more severe pre-treatment SI were less likely to achieve an SI response to a single ketamine infusion. On the other hand, according to our subgroup analysis, ketamine infusions were effective in reducing SI overall even among those with marked SI. In the present study, participants had an initial reduction in $\mathrm{SI}$ with the single Phase 1 infusion that persisted despite relapse of depressive symptoms and further decreased with repeated infusions administered during Phase 2 . This carryover effect suggests that the antisuicidal effects of ketamine may last longer than its antidepressant effects, though this was not directly tested. According to clinician-administered MADRS-SI scores, SI decreased significantly after three Phase 2 infusions while with self-reported QIDS-SI scores, the full course of six infusions was required to result in a significant $\mathrm{SI}$ decrease. This could be due to a difference in the measures. Nevertheless, participants obtained a decrease in SI following successive Phase 2 infusions, with both measures suggesting cumulative effects. Consequently, patients may gain additional benefits from repeated infusions beyond those obtained from single infusions. Within the present sample, a doubling of antidepressant response rates was achieved with repeated infusions [19].

During Phase 2, participants received ketamine infusions three times per week. While other studies have administered repeated infusions with similar frequency $[18,31,32]$, the optimal administration schedule to maximally reduce $S I$ has yet to be determined. A recent randomized controlled trial that administered ketamine twice a week for 3 weeks found no difference between ketamine and saline placebo on SI or depressive symptoms [33]. In that trial, participants were selected for severe, resistant depression with chronic $\mathrm{SI}$ and the authors suggest that the dose administered $(0.5 \mathrm{mg} / \mathrm{kg})$ may be insufficient for that patient population. Most studies reporting reduced SI with ketamine, including the present study, have administered ketamine at that dose. While Cusin et al. [34] reported improved antidepressant response to repeated ketamine in patients using a two-step dose-escalation strategy; this dosing strategy was not shown to affect SI response [16]. Further examination of the effects of variable dosing schedules and dose-escalation strategies on change in $\mathrm{SI}$ is warranted to identify optimal administration parameters to maximize patient response.

The present findings suggest that the effects of ketamine on $\mathrm{SI}$ and depression are partially independent. This is supported by the facts that $60 \%$ of the variance in overall change in SI was not explained by change in depressive symptoms. Participants' change in SI with ketamine remained significant when adjusted for their change in depressive symptom scores, and several individuals who failed to meet antidepressant response criteria with ketamine still experienced an alleviation of SI with repeated infusions. These results are consistent with the findings of a metaanalysis that reported that the effects of ketamine on SI are partially independent from its effects on depressive symptoms [7]. This partial independence hints at the potential use of ketamine as a treatment for SI outside the context of TRD.

As per the study design, participants who obtained an antidepressant response to ketamine following the course of six repeated infusions received an additional four infusions administered once per week. Weekly infusions were sufficient to sustain reductions of $\mathrm{SI}$ in responders during this treatment prolongation. This finding has important clinical relevance as it suggests the frequency of ketamine administration can be reduced once patients have obtained a reduction in SI and beneficial effects can be maintained. The optimal frequency of maintenance infusions to prolong the suppression of SI obtained with repeated infusions however remains unknown. While the antidepressant effects of ketamine last an average of 18-19 days [22,31], there is a wide variability among individual patients. Thus far, the duration of ketamine's antisuicidal effects have not been well described. Grunebaum et al. [14] reported sustained reduction of SI for 6 weeks following a single infusion in a non-resistant MDD sample. More formal follow-up of the duration of persisting SI effects of ketamine treatment is required to permit tailoring of maintenance infusions and to better assess returning suicide risk among patients. 
Despite the strengths of this study, limitations should be considered. First, this study was a secondary analysis of a clinical trial designed to evaluate the efficacy of the antidepressant effects of ketamine. Since the Phase 1 double-blind crossover was timed to capture participants' relapse in depressive symptoms, not SI, there was a significant carryover of the initial decrease in $\mathrm{SI}$ in subjects randomized to receive ketamine prior to midazolam. Ideally, SI would have had to return to baseline levels prior to participants crossing over to the second medication but SI was not the primary study outcome. A second limitation is the absence of MADRS-SI assessment between $24 \mathrm{~h}$ and 7 days postinfusion in Phase 1. However, carryover of antisuicidal effects into Phase 2 of the study despite relapse of depressive symptoms suggests that significant findings at 7 days postinfusion are not spurious.

Third, repeated and maintenance infusions were administered open-label without a control condition. Since participants were exposed to both ketamine and midazolam during Phase 1, the use of a control in subsequent phases would not have been successful as most participants could readily discriminate ketamine from midazolam after receiving both medications due to its mild dissociative effects [19]. Fourth, all study participants had TRD; therefore the results may not generalize to other patients with MDD, or other individuals with SI. However, reports of reduction of acute SI with ketamine treatment in post-traumatic stress disorder $[15,35]$, active military personnel [36], and patients newlydiagnosed with cancer [37], suggest the potential use of ketamine for suicidality beyond mood disorders. Fifth, SI was primarily assessed using the single item MADRS-SI. Although this clinicianrated measure significantly correlated with the patient-rated QIDS$\mathrm{SI}$, future studies would benefit from the inclusion of more comprehensive measures of suicidality (such as the Columbia Suicide Severity Rating Scale [38]) as well as examination of its potential contributing components (i.e., hopelessness, impulsivity, etc.). Sixth, given the limited sample size in the present study the results should be interpreted with caution. Lastly, while the longterm side effects of ketamine are currently unknown, the biological burden of prolonged depression and associated risk of suicide may outweigh the eventual identification of protracted side effects. This risk-benefit ratio has to be considered in the light that an anesthetic dose of ketamine produces a plasma level ten times higher than that used herein [12].

In summary, this study showed that single and repeated ketamine infusions reduced $\mathrm{SI}$ in individuals with TRD, with cumulative effects observed with thrice-weekly repeated infusions, and maintenance of the effects of ketamine on SI with once-weekly infusions. Current treatment options for $\mathrm{SI}$ are limited and these findings add to the growing body of research suggesting the promise of ketamine as a novel treatment strategy for SI. In particular, the preliminary evidence of rapid reduction in SI with ketamine suggests its use as a possible emergency strategy for individuals at high risk of suicide as an alternative to more disruptive and costly hospitalization. How long the antisuicidal effects of repeated ketamine infusions last, and whether ketamineinduced reduction in $\mathrm{SI}$ can be maintained with alternate pharmacological strategies remains unknown. Moreover, further research is required to determine whether reductions in $\mathrm{SI}$ following ketamine treatment translate into decreased risk for suicidal behavior and suicide attempts.

\section{FUNDING AND DISCLOSURE}

This work was supported by the Canadian Institutes of Health Research (to PB). Dr. Blier has received research support or speakers honoraria from, or has served as a consultant to, Allergan, Bristol Myers Squibb, Janssen, Lundbeck, Otsuka, Pierre Fabre Médicaments, Pfizer, Sunovion, and Takeda. The other authors declare no competing interests.

\section{ACKNOWLEDGEMENTS}

The authors thank Wendy Fusee, Holly English, Maria da Silva, Sara de la Salle, Hassan Khan, and Drs. Mostafa El Mansari, and Jean Blier for their contributions.

\section{ADDITIONAL INFORMATION}

Supplementary Information accompanies this paper at (https://doi.org/10.1038/ s41386-019-0570-x).

Publisher's note Springer Nature remains neutral with regard to jurisdictional claims in published maps and institutional affiliations.

\section{REFERENCES}

1. Coryell W, Young EA. Clinical predictors of suicide in primary major depressive disorder. Clin Psychiatry. 2005;66:412-17.

2. Rush AJ. Limitations in efficacy of antidepressant monotherapy. J Clin Psychiatry. 2007;68:8-10.

3. Pfeiffer PN, Hyungjin MK, Ganoczy D, Zivin K, Valenstein M. Treatment-resistant depression and risk of suicide. Suicide Life-Threat Behav. 2013;43:356-65.

4. Klonsky ED, May AM, Saffer BY. Suicide, suicide attempts, and suicidal ideation. Annu Rev Clin Psychol. 2016;12:307-30.

5. Griffiths JJ, Zarate CA Jr, Rasimas JJ. Existing and novel biological therapeutics in suicide prevention.Am J Prev Med. 2014;47:S195-203.

6. Sanacora G, Frye MA, McDonald W, Mathew SJ, Turner MS, Schatzberg AF, et al. A consensus statement on the use of ketamine in the treatment of mood disorders. JAMA Psychiatry. 2017;74:399-405

7. Wilkinson ST, Ballard ED, Bloch MH, Mathew SJ, Murrough JW, Feder A, et al. The effect of a single dose of intravenous ketamine on suicidal ideation: a systematic review and individual participant data meta-analysis. Am J Psychiatry. 2018;175:150-58.

8. Holtz A, Mülsch F, Schwarz MK, Hollmann M, Döbrössy MD, Coenen VA, et al Enhanced $m$ Glu5 signaling in excitatory neurons promotes rapid antidepressant effects via AMPA receptor activation. Neuron. 2019;104:1-15.

9. Ballard ED, lonescu DF, Vande Voort JL, Niciu MJ, Richards EM, Luckenbaugh DA et al. Improvement in suicidal ideation after ketamine infusion: relationship to reductions in depression and anxiety. J Psychiatr Res. 2014;58:161-66.

10. Price RB, losifescu DV, Murrough JW, Chang LC, Al Jurdi RK, Iqbal SZ, et al. Effects of ketamine on explicit and implicit suicidal cognition: a randomized controlled trial in treatment-resistant depression. Depress Anxiety. 2014;31:335-43.

11. Vande Voort JL, Ballard ED, Luckenbaugh DA, Bernert RA, Richards EM, Niciu MJ, et al. Antisuicidal response following ketamine infusion is associated with decreased nighttime wakefulness in major depressive disorder and bipolar disorder. J Clin Psychiatry. 2017;78:1068-74.

12. Zarate CA Jr, Brutsche NE, Ibrahim L, Franco-Chaves J, Diazgranados N, Cravchik A, et al. Replication of ketamine's antidepressant efficacy in bipolar depression: a randomized controlled add-on trial. Biol Psychiatry. 2012;71:939-46.

13. DiazGranados N, Ibrahim LA, Brutsche NE, Ameli R, Henter ID, Luckenbaugh DA, et al. Rapid resolution of suicidal ideation after a single infusion of an $\mathrm{N}$-methylD-aspartate antagonist in patients with treatment-resistant major depressive disorder. J Clin Psychiatry. 2010;71:1605-11.

14. Grunenbaum MF, Galfalvy HC, Choo TH, Keilp JG, Moitra VK, Parris MS, et al. Ketamine for rapid reduction of suicidal thoughts in major depression: a midazolam-controlled randomized clinical trial. Am J Psychiatry. 2018;175:327-35.

15. Murrough JW, Soleimani L, DeWilde KE, Collins KA, Lapidus KA, lacoviello BM, et al. Ketamine for rapid reduction of suicidal ideation: a randomized controlled trial. Psychol Med. 2015;45:3571-80.

16. Ionescu DF, Swee MB, Pavone KJ, Taylor N, Akeju O, Baer L, et al. Rapid and sustained reductions in current suicidal ideation following repeated doses of intravenous ketamine: secondary analysis of an open-label study. J Clin Psychiatry. 2016;77:e719-25.

17. Vande Voort JL, Morgan RJ, Kung S, Rasmussen KG, Rico J, Palmer BA, et al. Continuation phase intravenous ketamine in adults with treatment-resistant depression. J Affect Disord. 2016;206:300-4.

18. Zheng W, Zhou Y-L, Liu W-J, Wang C-Y, Zhan Y-N, Li H-Q, et al. Rapid and longerterm antidepressant effects of repeated-dose intravenous ketamine for patients with unipolar and bipolar depression. J Psychiatr Res. 2018;106:61-8.

19. Phillips JL, Norris S, Talbot J, Birmingham M, Hatchard T, Ortiz A, et al. Single, repeated, and maintenance ketamine infusions for treatment-resistant depression: a randomized controlled trial. Am J Psychiatry. 2019;176:401-9.

20. Ballard ED, Yarrington JS, Farmer CA, Richards E, Machado-Vieira R, Kadriu B, et al. Characterizing the course of suicidal ideation response to ketamine. J Affect Disord. 2018;241:86-93. 
21. Yanni Z, Bin Z, Yanling Z, Wei Z, Weijian L, Chengyu W, et al. A preliminary study of anti-suicidal efficacy of repeated ketamine infusions in depression with suicidal ideation. J Affect Disord. 2019;251:205-12.

22. Murrough JW, Perez AM, Pillemer S, Stern J, Parides MK, aan het Rot $M$, et al. Rapid and longer-term antidepressant effects of repeated ketamine infusions in treatment-resistant major depression. Biol Psychiatry. 2013;74:250-6.

23. American Psychiatric Association. Diagnostic and statistical manual of mental disorders. 4th ed. Washington, DC: American Psychiatric Association; 2000.

24. Sheehan DV, Lecrubier $Y$, Sheehan KH, Amorim P, Janavs J, Weiller E, et al. The Mini-International Neuropsychiatric Interview (M.I.N.I.): the development and validation of a structured diagnostic psychiatric interview for DSM-IV and ICD-10. J Clin Psychiatry. 1998;59(Suppl 20):22-33.

25. Montgomery SA, Åsberg M. A new depression scale designed to be sensitive to change. Br J Psychiatry. 1979;134:382-9.

26. Sackeim HA. The definition and meaning of treatment-resistant depression. J Clin Psychiatry. 2001;62(Suppl 16):10-7.

27. Frye MA, Blier $\mathrm{P}$, Tye SJ. Concomitant benzodiazepine use attenuates ketamine response: implications for large scale study design and clinical development. J Clin Psychopharmacol. 2015;35:334-6.

28. Rush AJ, Trivedi MH, Ibrahim HM, Carmody TJ, Arnow B, Klein DN, et al. The 16Item Quick Inventory of Depressive Symptomatology (QIDS), clinician rating (QIDS-C), and self-report (QIDS-SR): a psychometric evaluation in patients with chronic major depression. Biol Psychiatry. 2003;54:573-83.

29. Ballard ED, Luckenbaugh $D A$, Richards EM, Walls $T L$, Brutsché $N E$, Ameli $R$, et al. Assessing measures of suicidal ideation in clinical trials with a rapid-acting antidepressant. J Psychiatr Res. 2015;68:68-73.

30. Akaike H. A new look at the statistical model identification. IEEE Trans Autom Contr. 1974:19:716-23.
31. aan het Rot M, Collins KA, Murrough JW, Perez AM, Reich DL, Charney DS, et al. Safety and efficacy of repeated-dose intravenous ketamine for treatmentresistant depression. Biol Psychiatry. 2010;67:139-45.

32. Sinyor $M$, Williams $M$, Belo $S$, Orser B, Vincent $M$, Mah $L$, et al. Ketamine augmentation for major depressive disorder and suicidal ideation: preliminary experience in an inpatient psychiatry setting. J Affect Disord. 2018;241:103-9.

33. Ionescu DF, Bentley KH, Eikermann M, Taylor N, Akeju O, Swee MB, et al. Repeatdose ketamine augmentation for treatment-resistant depression with chronic suicidal ideation: a randomized, double blind, placebo controlled trial. J Affect Disord. 2019;243:516-24.

34. Cusin C, lonescu DF, Pavone KJ, Akeju O, Cassano P, Taylor N, et al. Ketamine augmentation for outpatients with treatment-resistant depression: preliminary evidence for two-step intravenous dose escalation. Aust N. Z J Psychiatry. 2017;51:55-64.

35. Feder A, Parides MK, Murrough JW, Perez AM, Morgan JE, Saxena S, et al. Efficacy of intravenous ketamine for treatment of chronic posttraumatic stress disorder: a randomized clinical trial. JAMA Psychiatry. 2014;71:681-8.

36. Burger J, Capobianco M, Lovern R, Boche B, Ross E, Darracq MA, et al. A doubleblinded, randomized, placebo-controlled sub-dissociative dose ketamine pilot study in the treatment of acute depression and suicidality in a military emergency department setting. Mil Med. 2016;181:1195-9.

37. Fan W, Yang $H$, Sun $Y$, Zhang J, Li G, Zheng $Y$, et al. Ketamine rapidly relieves acute suicidal ideation in cancer patients: a randomized controlled clinical trial. Oncotarget. 2017;8:2356-60.

38. Posner K, Brown GK, Stanley B, Brent DA, Yershova KV, Oquendo MA, et al. The Columbia-Suicide Severity Rating Scale: initial validity and internal consistency findings from three multisite studies with adolescents and adults. Am J Psychiatry. 2011;168:1266-77. 